\title{
BMJ Open Sleep among bereaved caregivers of patients admitted to hospice: a 1-year longitudinal pilot study
}

\author{
Anners Lerdal, ${ }^{1,2}$ Kari Slåtten, ${ }^{3}$ Elisabeth Saghaug, ${ }^{2}$ Ellen Karine Grov, ${ }^{4,5}$ \\ Are Peder Normann, ${ }^{2}$ Kathryn A Lee, ${ }^{6}$ Bjørn Bjorvatn, ${ }^{7,8}$ Caryl L Gay, ${ }^{2,6}$
}

To cite: Lerdal A, Slåtten $K$, Saghaug $\mathrm{E}$, et al. Sleep among bereaved caregivers of patients admitted to hospice: a 1-year longitudinal pilot study. BMJ Open 2016;6: e009345. doi:10.1136/ bmjopen-2015-009345

- Prepublication history for this paper is available online. To view these files please visit the journal online (http://dx.doi.org/10.1136/ bmjopen-2015-009345).

Received 8 July 2015 Accepted 2 November 2015

CrossMark

For numbered affiliations see end of article.

Correspondence to Dr Anners Lerdal; anners. lerdal@medisin.uio.no

\section{ABSTRACT}

Objectives: This pilot study aimed to describe the sleep of partners and other family caregivers prior to and in the first year after a hospice patient's death. The study also evaluated the feasibility of the study protocol and determined the effect sizes in preparation for a full-scale study.

Design: The pilot study used a longitudinal, descriptive and comparative design.

Setting and participants: Participants included primary family caregivers of patients admitted to a hospice in Oslo, Norway.

Primary outcome: Caregiver sleep was measured subjectively with the Pittsburgh Sleep Quality Index (PSQI) and objectively using wrist actigraphy for 4 nights and 3 days at three different times: during the hospice stay, and at 6 and 12 months after the patient's death.

Results: 16 family caregivers (10 partners and 6 other family members) completed the 1-year study protocol. Overall, sleep quality and quantity were stable over time and at each assessment, approximately half of the sample had poor sleep quality, both by self-report and objective measures. However, the sleep trajectories differed significantly over time, with older caregivers ( $\geq 65$ years) having significantly longer sleep durations than younger caregivers ( $<65$ years). Furthermore, sleep quality also differed over time depending on the caregiver's relationship to the patient, with partner caregivers having significantly worse sleep quality than other family caregivers.

Conclusions: Caring for a dying family member is known to interfere with sleep, yet little is known about bereaved caregivers. The results of this pilot study demonstrate the feasibility of the longitudinal study protocol and indicate that sleep problems are common for caregivers and continue into the bereavement period, particularly for partner caregivers. The caregiver's relationship to the patient may be an important factor to consider in future studies.

\section{INTRODUCTION}

Sleep disturbance is a common and often distressing symptom for caregivers, particularly caregivers who provide end-of-life

\section{Strengths and limitations of this study}

- A strength of the study is the use of both objective and subjective measures of sleep.

- The inclusion of sleep measures before and after the patient's death is also a strength.

- While the findings indicate a high prevalence of sleep disturbance among caregivers, particularly partners, during the first year of bereavement the small sample size does not allow generalisation of the study findings.

- The $50 \%$ enrolment rate and the $20 \%$ attrition rate may limit the representativeness of the sample, and suggest that strategies for improving participant enrolment and retention may need further consideration in a full-scale study of bereaved caregivers.

- The patients and caregivers were recruited while receiving care at a hospice, and the findings may differ for caregivers of patients receiving home care service.

care. ${ }^{1-5}$ Inadequate sleep may lead to poorer long-term outcomes and stress-related disorders. ${ }^{6}$ Prior studies have reported that some groups of caregivers may be vulnerable to developing sleep disturbances due to older age, male gender, role overload and depressive symptoms. ${ }^{7}$ During the bereavement period, women have been reported to experience more severe anxiety than bereaved men, although gender was not associated with prolonged grief or depression. ${ }^{8}$ The caregiver's relationship to the patient (ie, partner or other family member) has not been associated with sleep disturbance during the caregiving period in several prior studies, ${ }^{129}$ but to the best of our knowledge, it has not been examined during the bereavement period.

Few longitudinal studies have evaluated sleep across the transition from end-of-life caregiving into the bereavement period. ${ }^{7}{ }^{10}$ Nonetheless, this transition may be a critical health risk factor for caregivers adjusting to 
the loss of their loved one. Furthermore, sleep during this period may play an important role in health, as sleep disturbance can exacerbate existing distress, whereas adequate sleep can foster optimal physical, mental and social health.

The purpose of this pilot study was to examine the feasibility of conducting a longitudinal sleep study of bereaved family caregivers for patients in hospice and describe the sleep trajectories of family caregivers from the hospice period until 1 year after the patient's death. We hypothesised that family caregivers would have high rates of clinically significant sleep disturbance (based on objective and subjective measures), not only during the caregiving period but into the bereavement period as well, and that partners would have worse sleep trajectories than other family caregivers.

\section{METHODS}

This study reports the findings from a longitudinal pilot study of caregivers for patients admitted to Lovisenberg Hospice in Oslo, Norway. Data were collected from January 2012 to February 2014 from hospice patients and their primary caregivers; only data from caregivers are included in this report. Caregivers were also assessed 6 and 12 months after the patient's death. Informed written consent was obtained from all patients and their caregivers.

\section{Participants}

Consecutive patients were recruited if they met the following inclusion criteria: (1) admitted for 24-h hospice care within the past week, (2) evaluated by their health professional to be in the terminal stage of their disease, (3) $\geq 18$ years of age, (4) could specify a primary caregiver and (5) had the cognitive and verbal capacity to comprehend Norwegian verbal cues. All hospice patients had advanced cancer. Each patient who consented to participate specified their primary caregiver who was also recruited for participation. Caregivers who were $\geq 18$ years of age and consented to participate were included (figure 1). Sixteen family caregivers (10 partners and 6 other family members) completed the 1 -year study protocol.

\section{Measurements}

Perceived sleep quality

Caregivers' sleep quality during the prior month was assessed with the 19-item Pittsburgh Sleep Quality Index (PSQI) ${ }^{11}$ Total scores range from 0 to 21 , where higher scores represent poorer sleep quality, and scores $>5$ are considered clinically significant. ${ }^{11}$ The PSQI has seven components: sleep quality, sleep latency, sleep duration, habitual sleep efficiency, sleep disturbance, sleep medication use and daytime dysfunction. Component scores range from 0 to 3, with higher scores reflecting worse sleep. Internal consistency and test-retest reliability for the original ${ }^{12}$ and Norwegian versions are satisfactory. ${ }^{13}$

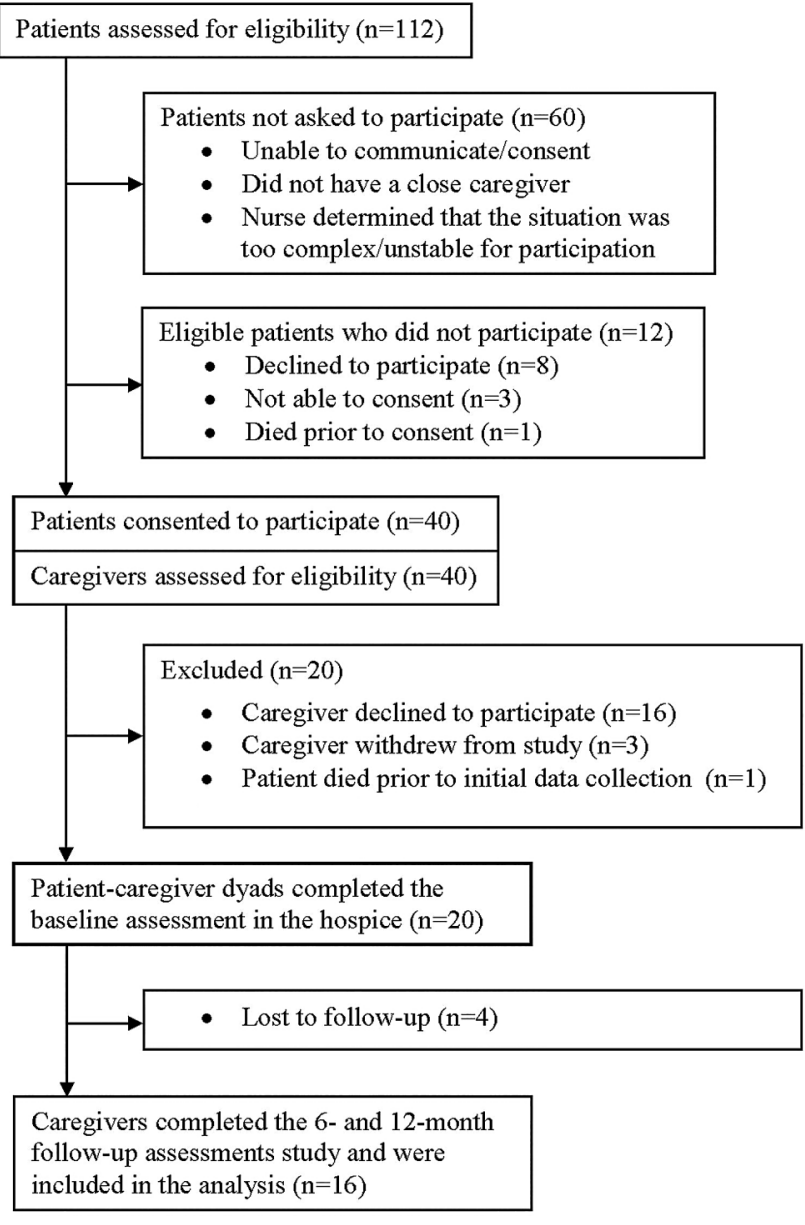

Figure 1 Flowchart showing the recruitment of patient-caregiver dyads.

\section{Sleep-wake pattern}

Sleep quantity and sleep disruption were estimated with the Motionlogger Actigraph (AA-32 Ambulatory Monitoring Inc, Ardsley, New York, USA), a valid and reliable instrument for assessing sleep-wake behaviour over several nights and days. ${ }^{14}$ It is worn like a wristwatch and records movement in $1 \mathrm{~min}$ intervals to estimate sleep and wake time. Its correspondence with polysomnography is $91-93 \%$ in healthy people. ${ }^{14}$ In this study, the actigraph was worn for 4 nights and 3 days. Actigraphs recorded in zero-crossing mode, and activity counts were analysed with the Cole-Kripke algorithm from ActionW software program, V.2.4 (Ambulatory Monitoring Inc). Sleep diaries facilitated actigraphy scoring for bedtime and final wake time. Night-time sleep parameters were based on standard intervals from 21:00 to 08:59, and included measures of both sleep quantity (total night-time sleep duration) and sleep disruption (mean number of awakenings and mean wake after sleep onset (WASO)). WASO was reported as the number of minutes spent awake after initially falling asleep, as well as a percentage standardised by time in bed. WASO $>30 \mathrm{~min}$ is considered clinically significant and suggestive of maintenance insomnia. Daytime sleep 
Table 1 Sociodemographic characteristics for the pilot sample of caregiver's relationship to the patient $(\mathrm{N}=16)$

\begin{tabular}{|c|c|c|c|c|c|}
\hline Sociodemographic characteristics & $\begin{array}{l}\text { Total } \\
\mathrm{N}=16\end{array}$ & $\begin{array}{l}\text { Partners } \\
n=10\end{array}$ & $\begin{array}{l}\text { Other family } \\
n=6\end{array}$ & p Value & Effect size \\
\hline \multicolumn{6}{|l|}{ Caregiver characteristics } \\
\hline Age (years), mean (SD) & $58.6(13.8)$ & $65.1(9.9)$ & $47.7(11.3)$ & $0.009^{*}$ & $\eta^{2}=0.399$ \\
\hline Range & $36-77$ & $46-77$ & $36-72$ & & \\
\hline Gender, n (\%) & & & & $0.14 \dagger$ & $\phi=0.372$ \\
\hline Male & $3(19)$ & $3(30)$ & $0(0)$ & & \\
\hline Female & $13(81)$ & $7(70)$ & $6(100)$ & & \\
\hline Education, years & & & & $>0.99 \dagger$ & $\phi=0.067$ \\
\hline$\leq 13$ & $6(38 \%)$ & $4(40 \%)$ & $2(33 \%)$ & & \\
\hline 14 or more & $10(62 \%)$ & $6(60 \%)$ & $4(67 \%)$ & & \\
\hline Work status & & & & $0.12 \dagger$ & $\phi=0.516$ \\
\hline In paid job & $8(50 \%)$ & $3(30 \%)$ & $5(83 \%)$ & & \\
\hline Not in paid job & $8(50 \%)$ & 7 (70\%) & $1(17 \%)$ & & \\
\hline Relationship status & & & & $0.38 \dagger$ & $\phi=0.333$ \\
\hline In paired relationship & $15(94 \%)$ & $10(100 \%)$ & $5(83 \%)$ & & \\
\hline Not in relationship & $1(6 \%)$ & - & $1(17 \%)$ & & \\
\hline \multicolumn{6}{|l|}{ Relationship to patient $\ddagger$} \\
\hline Partner/spouse & $10(60 \%)$ & $10(100 \%)$ & & & \\
\hline Child & $4(25 \%)$ & & $4(66 \%)$ & & \\
\hline Parent & $1(10 \%)$ & & $1(17 \%)$ & & \\
\hline Sibling & $1(5 \%)$ & & $1(17 \%)$ & & \\
\hline \multicolumn{6}{|l|}{ Patient characteristics } \\
\hline Age (years), mean (SD) & $69.1(9.1)$ & $69.5(10.6)$ & $68.3(6.7)$ & $0.81 \S$ & $\eta^{2}=0.004$ \\
\hline Range & $36-84$ & $46-84$ & $36-72$ & & \\
\hline Gender, n (\%) & & & & $0.034 \dagger$ & $\phi=0.600$ \\
\hline Male & $6(38)$ & $6(60)$ & - & & \\
\hline Female & $10(62)$ & $4(40)$ & $6(100)$ & & \\
\hline
\end{tabular}

Statistically significant differences are indicated in bold type.

${ }^{*} \mathrm{~F}(1,14)=9.29$.

†Fisher's Exact.

$\ddagger$ No analyses since groups differ by definition.

$\S \mathrm{F}(1,14)=0.06$.

quantity was estimated as the number of min spent asleep between 09:00 and 20:59. Total sleep quantity during each $24 \mathrm{~h}$ period was calculated as the sum of night and day sleep, and mean values were calculated across the first three $24 \mathrm{~h}$ periods. As bedtimes and wake times were only roughly estimated in the sleep diaries, sleep onset latency and sleep efficiency were not included in this study.

\section{Data analysis}

An initial sample size of 20 caregivers was considered sufficient for determining the protocol's feasibility (including enrolment and retention rates) and estimating effect sizes for change over time, and for differences between partners and other family caregivers. Data were analysed using SPSS V.22.0. Descriptive statistics summarised demographic characteristics and sleep outcome variables. Group differences were assessed using Fisher's exact test and analysis of variance. In separate analyses evaluating age group (age $<65$ or $\geq 65$ years) and caregiver group (partner or other), repeated measures analysis of variance was used to evaluate the effects of group, time (in hospice, and at 6 and 12 months follow-up), and the interaction of group by time. Relevant covariates were also included in the models. Significance of $\mathrm{p}<0.05$ was used for all analyses. Effect sizes of $\eta^{2}$ values $>0.04$ and $\phi$ values $>0.2$ were considered clinically relevant. ${ }^{15}$

\section{RESULTS}

Descriptive statistics

As shown in figure 1, the enrolment rate was $50 \%$ and the attrition rate was $20 \%$. Sixteen caregivers completed all three assessments and were included in the analysis (figure 1). Sociodemographic characteristics are shown in table 1. Partners $(n=10)$ were older on average compared to other family caregivers $(n=6)$. Most patients were female $(n=10)$, and the six male patients were all cared for by their female partners, resulting in a significant association between patient gender and the caregiver's relationship to the patient (partner vs other family member). Although not statistically significant in this small sample, there were clinically relevant group differences in caregiver gender and work status.

\section{Sleep measures over time}

Mean PSQI scores for each assessment are reported in table 2. Scores generally indicated significant sleep 
Table 2 Sleep measures over time, mean (SD), N=16

\begin{tabular}{|c|c|c|c|}
\hline Sleep measure & $\begin{array}{l}\text { In } \\
\text { hospice }\end{array}$ & 6 Months & 12 Months \\
\hline \multicolumn{4}{|c|}{ Self-reported sleep quality } \\
\hline PSQI total score & $6.56(3.10)$ & $5.81(3.35)$ & $5.94(2.57)$ \\
\hline PSQI sleep quality & $0.44(1.03)$ & $0.38(0.89)$ & $0.25(0.77)$ \\
\hline $\begin{array}{l}\text { PSQI sleep onset } \\
\text { latency }\end{array}$ & $1.13(0.74)$ & $1.00(0.89)$ & $0.94(1.12)$ \\
\hline $\begin{array}{l}\text { PSQI sleep } \\
\text { duration }\end{array}$ & $0.50(0.82)$ & $0.63(0.89)$ & $0.81(0.83)$ \\
\hline $\begin{array}{l}\text { PSQI habitual } \\
\text { sleep efficiency }\end{array}$ & $0.88(0.96)$ & $1.13(1.20)$ & $0.94(1.18)$ \\
\hline $\begin{array}{l}\text { PSQI sleep } \\
\text { disturbance }\end{array}$ & $1.44(0.51)$ & $1.31(0.60)$ & $1.25(0.45)$ \\
\hline $\begin{array}{l}\text { PSQI sleep } \\
\text { medication* }\end{array}$ & $0.56(0.81)$ & $0.13(0.34)$ & $0.25(0.45)$ \\
\hline $\begin{array}{l}\text { PSQI daytime } \\
\text { dysfunction }\end{array}$ & $1.69(0.95)$ & $1.25(0.86)$ & $1.50(0.82)$ \\
\hline \multicolumn{4}{|l|}{ Actigraph measures } \\
\hline \multicolumn{4}{|l|}{ Sleep disruption } \\
\hline $\begin{array}{l}\text { Night } \\
\text { awakenings, } n\end{array}$ & $7.13(4.39)$ & $8.96(5.14)$ & $8.68(5.02)$ \\
\hline WASO, min & $105(80)$ & $87(48)$ & $89(54)$ \\
\hline WASO, \% & $16.7(10.8)$ & $14.9(7.0)$ & $15.4(9.5)$ \\
\hline \multicolumn{4}{|l|}{ Sleep quantity } \\
\hline $\begin{array}{l}\text { Total sleep in } \\
24 \mathrm{~h}, \mathrm{~h}\end{array}$ & $8.30(1.29)$ & $8.16(1.18)$ & $8.60(1.64)$ \\
\hline $\begin{array}{l}\text { Total night } \\
\text { sleep, } \mathrm{h}\end{array}$ & $7.73(1.10)$ & $7.64(0.80)$ & $7.78(1.13)$ \\
\hline $\begin{array}{l}\text { Total day sleep, } \\
\text { min }\end{array}$ & $33.2(28.9)$ & $30.9(28.1)$ & $48.8(43.4)$ \\
\hline
\end{tabular}

PSQI total scores $>5$ are clinically significant and component scores range is $0-3$, with higher scores indicating poorer sleep. The PSQI assessed sleep during past month, while actigraphy measures assessed average sleep over 4 nights and 3 days. There were no significant changes over time for any sleep measure, except the PSQI sleep medication component. ${ }^{*} \mathrm{~F}=4.27, \mathrm{p}=0.042$ (Greenhouse-Geisser correction), $\eta^{2}=0.22$. PSQI, Pittsburgh Sleep Quality Index; WASO, wake after sleep onset, a measure of sleep disruption. disturbance, and at each of the three assessments-in hospice, at 6 and 12 months follow-up-approximately half of the caregivers scored above the PSQI clinical cut-off of $5(62.5 \%, 43.8 \%$ and $50 \%$, respectively). Repeated measures analysis indicated that PSQI scores were stable across the three assessments, with no significant effect of time. The PSQI component scores indicated that difficulty falling asleep, waking up during the night, and daytime dysfunction were the most commonly reported sleep problems. The component scores were also stable over time, with only the sleep medication component scores improving (ie, scores decreased) significantly over time.

Objective measures of sleep indicated significant sleep disruption at each of the three assessments, and all but two of the caregivers had WASO $>30 \mathrm{~min}$. With respect to sleep quantity, the mean night-time sleep duration was 7.5-8 h, well within the recommended range. Caregivers also had an average of $30 \mathrm{~min}$ of daytime napping, such that their average sleep time over $24 \mathrm{~h}$ exceeded $8 \mathrm{~h}$ at each assessment.

\section{Sleep trajectories by caregiver characteristics}

To determine the effect of the caregiver's age on their sleep trajectories from the hospice period until 1 year after the patient's death, a repeated measures analysis of variance was conducted, with caregiver age included as a covariate. In these analyses, age was a significant factor for both night-time sleep $(\mathrm{F}[1,14]=11.2, \quad \mathrm{p}=0.005$, $\left.\eta^{2}=0.44\right)$ and total sleep in $24 \mathrm{~h}(\mathrm{~F}[1,14]=7.05, \mathrm{p}=0.019$, $\left.\eta^{2}=0.34\right)$. To illustrate the influence of caregiver age, the sample was split into those $<65$ and $\geq 65$ years, and the sleep trajectories for night-time sleep duration in the two age groups are shown in figure 2. Given the small number of men in this sample $(n=3)$, the effect of caregiver sex could not be reliably evaluated.
Figure 2 Night-time sleep duration over time by caregiver age group. Older caregivers obtained consistently more sleep at night compared to younger caregivers (effect of age group: $F$ $[1,14]=6.55, p=0.023, \eta^{2}=0.32$ ).

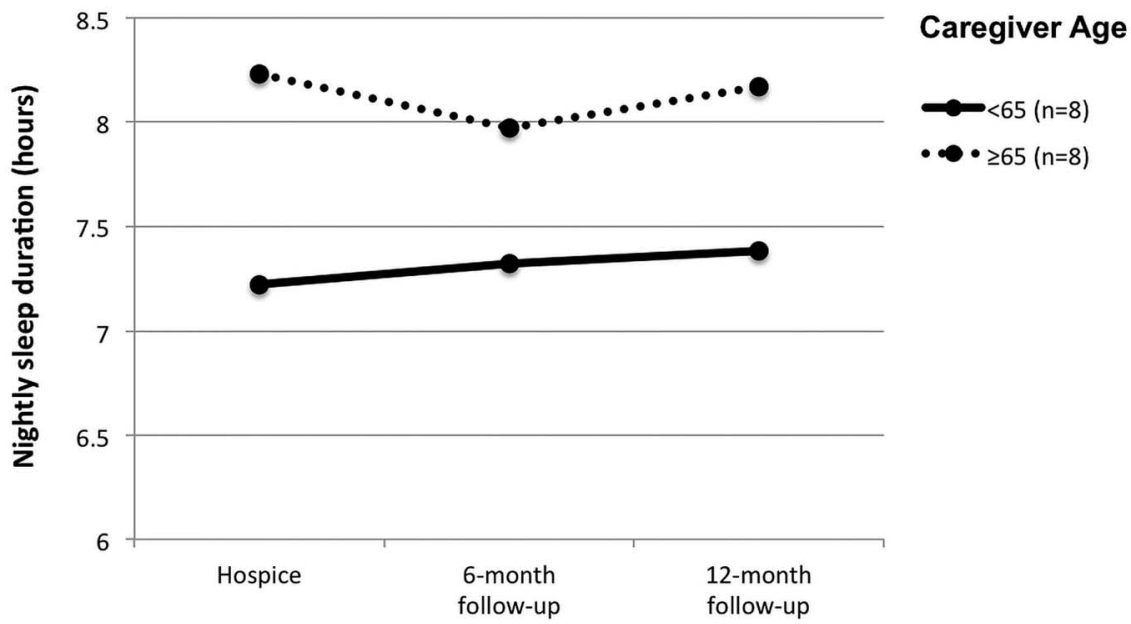

Time 
Table 3 Sleep measures over time by caregiver's relationship to patient and adjusted for caregiver age, mean $(95 \% \mathrm{Cl})$, $\mathrm{N}=16$

\begin{tabular}{|c|c|c|c|}
\hline Sleep measure & $\begin{array}{l}\text { Partners } \\
\mathrm{n}=10\end{array}$ & $\begin{array}{l}\text { Other family } \\
\mathrm{n}=6\end{array}$ & Statistics (with $p<0.10$ ) \\
\hline \multicolumn{4}{|l|}{ Self-reported sleep quality } \\
\hline PSQI total score & & & $p=0.040^{*}, \eta^{2}=0.29$ \\
\hline In hospice & 7.18 (4.70 to 9.65$)$ & 5.54 (2.14 to 8.94$)$ & \\
\hline 6-month follow-up & 7.45 (5.08 to 9.81$)$ & $3.09(-0.16$ to 6.34$)$ & \\
\hline 12-month follow-up & 7.69 (6.24 to 9.14$)$ & 3.02 (1.03 to 5.01$)$ & \\
\hline \multicolumn{4}{|l|}{ Actigraph measures } \\
\hline \multicolumn{4}{|l|}{ Sleep disruption } \\
\hline WASO, min & & & $p=0.002 \dagger, \eta^{2}=0.37$ \\
\hline In hospice & 70 (12 to 128$)$ & 165 (85 to 244$)$ & \\
\hline 6-month follow-up & 116 (86 to 147$)$ & $38(-4$ to 80$)$ & \\
\hline 12-month follow-up & 102 (60 to 144$)$ & 67 (9 to 124$)$ & \\
\hline WASO, \% & & & $p=0.007 \dagger, \eta^{2}=0.32$ \\
\hline In hospice & 12.0 (4.1 to 20.0$)$ & 24.5 (13.6 to 35.4$)$ & \\
\hline 6-month follow-up & 19.1 (14.5 to 23.6 ) & $7.9(1.7$ to 14.1$)$ & \\
\hline 12-month follow-up & 17.5 (10.0 to 25.0$)$ & $11.8(1.5$ to 22.1$)$ & \\
\hline Night awakenings, $\mathrm{n}$ & & & $p=0.067 \dagger, \eta^{2}=0.19$ \\
\hline In hospice & 7.1 (3.56 to 10.6$)$ & 7.2 (2.4 to 12.1$)$ & \\
\hline 6-month follow-up & 10.8 (6.8 to 14.7$)$ & 6.0 (0.6 to 11.3$)$ & \\
\hline 12-month follow-up & $10.3(6.5$ to 14.1$)$ & $6.0(0.7$ to 11.3$)$ & \\
\hline \multicolumn{4}{|l|}{ Sleep quantity } \\
\hline Total sleep in $24 \mathrm{~h}, \mathrm{~h}$ & & & - \\
\hline In hospice & 8.34 (7.44 to 9.25$)$ & 8.24 (7.00 to 9.48$)$ & \\
\hline 6-month follow-up & 8.49 (7.64 to 9.34$)$ & $7.61(6.44$ to 8.78$)$ & \\
\hline 12-month follow-up & 8.89 (7.75 to 10.0$)$ & 8.12 (6.55 to 9.69$)$ & \\
\hline Total night sleep, h & & & - \\
\hline In hospice & 7.80 (7.04 to 8.57$)$ & $7.61(6.56$ to 8.66$)$ & \\
\hline 6-month follow-up & 7.87 (7.30 to 8.44$)$ & 7.26 (6.48 to 8.05$)$ & \\
\hline 12-month follow-up & 7.83 (7.05 to 8.61$)$ & 7.68 (6.61 to 8.75$)$ & \\
\hline Total day sleep, min & & & - \\
\hline In hospice & 31.3 (8.0 to 54.7 ) & 36.4 (4.4 to 68.5$)$ & \\
\hline 6-month follow-up & 37.2 (15.5 to 58.9$)$ & $20.4(-9.5$ to 50.2$)$ & \\
\hline 12-month follow-up & 63.8 (31.9 to 95.8$)$ & $23.8(-20.1$ to 67.7$)$ & \\
\hline
\end{tabular}

The PSQI assessed sleep during past month, while actigraphy measures assessed average sleep over 4 nights and 3 days.

*Main effect for group (partner vs other family).

†Interaction effect for group by time.

PSQI, Pittsburgh Sleep Quality Index; WASO, wake after sleep onset, a measure of sleep disruption.

\section{Comparisons of partners and other family caregivers}

To evaluate whether partners and other family caregivers had similar sleep trajectories during the hospice period until 1 year after the patient's death, repeated measures analysis of variance was conducted for each sleep measure, with the caregiver's relationship to the patient included as the between-subjects factor. Given the difference in age between partners and other family caregivers, and the potential influence of age on sleep, the caregiver's age was also included as a covariate. Results indicated that partner caregivers reported consistently worse sleep quality than other family caregivers; although the self-reported sleep quality of other family caregivers improved over time, the interaction between time and caregiver type was not statistically significant $\left(p=0.195, \eta^{2}=0.12\right.$; table 3 and figure 3$)$. This pattern was also evident in the objective actigraphy measures of sleep disruption (WASO in minutes and as a per cent of time in bed), with partner caregivers having increasing levels of sleep disruption over time, while the sleep disruption of other family caregivers improved over time (figure 4). Although the effect did not reach statistical significance in this small sample, a similar pattern was observed for number of night awakenings and the effect size estimate suggests the effect may be clinically significant. There was no difference between partners and other family caregivers for sleep quantity based on actigraphy values; both types of caregivers obtained similar amounts of sleep over time.

\section{DISCUSSION}

Few longitudinal studies have evaluated sleep across the transition from end-of-life caregiving into the bereavement period. The results of this pilot study indicate that poor sleep quality is common among family caregivers 
Figure 3 Self-reported sleep quality over time by caregiver type, adjusting for caregiver age. Partner caregivers reported consistently worse sleep quality over time compared to other family caregivers (effect of caregiver type: $F[1,13]=5.18$, $\left.\mathrm{p}=0.040, \eta^{2}=0.29\right)$. Covariate of caregiver age evaluated at a mean value of 58.6 years.

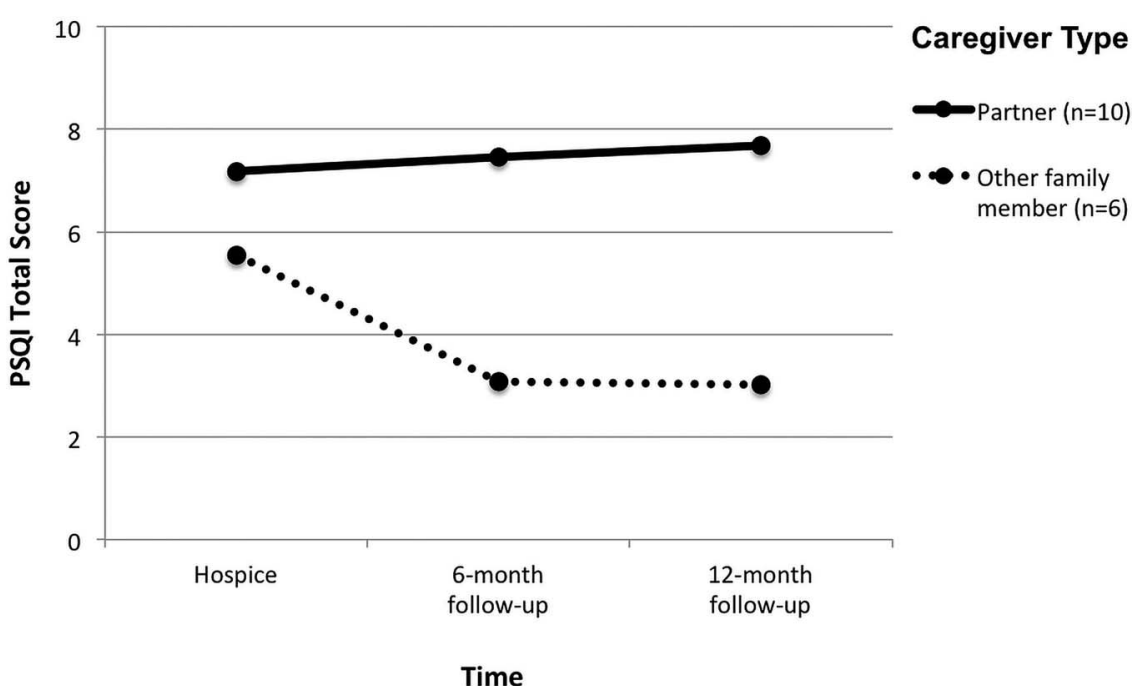

of patients in hospice and that their poor sleep continues through the first year of bereavement. However, sleep trajectories during this transition differ depending on at least two caregiver characteristics. First, older caregivers ( $\geq 65$ years) had significantly longer sleep durations than younger caregivers ( $<65$ years), although both groups generally obtain the recommended minimum sleep of $7 \mathrm{~h}$ per night. Second, partner caregivers reported consistently worse sleep disruption than other family caregivers, and objective actigraphy measures indicated that their sleep disruption actually worsened over time, while sleep improved for other family caregivers after the caregiving ended.

Partners had less sleep disruption (WASO) than other family caregivers after hospice admission (figure 4). However, sleep was less disrupted over time for other family caregivers and more disrupted for partner caregivers. These findings differ from prior studies that did not find differences by caregiver type. ${ }^{1}{ }^{9}$ The nonpartner caregivers in our sample were younger and more likely to be employed than the partner caregivers, and thus may have had work and other family responsibilities that interfered with sleep. It is also possible that the hospice admission allowed the partner to increase their sleep time for recovery from the chronic sleep debt experienced while the patient was at home.

Grov $^{16}$ points out that bereavement support is part of the palliative care philosophy. However, the bereavement process likely differs for partners compared to other family caregivers. While both adapt to a new life situation after the death of the patient, for partners this can mean completely redefining the focus of their life. ${ }^{17}$ According to Exley and Allen ${ }^{18}$ and Soothill et al, ${ }^{19}$ it is important to understand the impact of loss of a significant other on social identity and to note that 'home' is not merely a physical space, but the social and emotional relationships contained therein are also crucial.

Understanding the partner's future role when left alone requires important consideration of key variables such as understanding the role of family communication as it relates to end-of-life care. ${ }^{20}$ According to Kissane et $a l,{ }^{21-23}$ there is a large body of evidence from psychiatry that focuses on communication patterns and conflicts within families, and how these patterns and
Figure 4 Objectively measured sleep disruption over time by caregiver type, adjusting for caregiver age. Partners had less sleep disruption than other family caregivers while the patient was in hospice, but the sleep disruption improved over time for other family caregivers and worsened for partner caregivers (interaction effect of time by caregiver type: $F[2,26]=7.76$, $\left.p=0.002, \eta^{2}=0.37\right)$. Covariate of caregiver age evaluated as a mean value of 58.6 years.

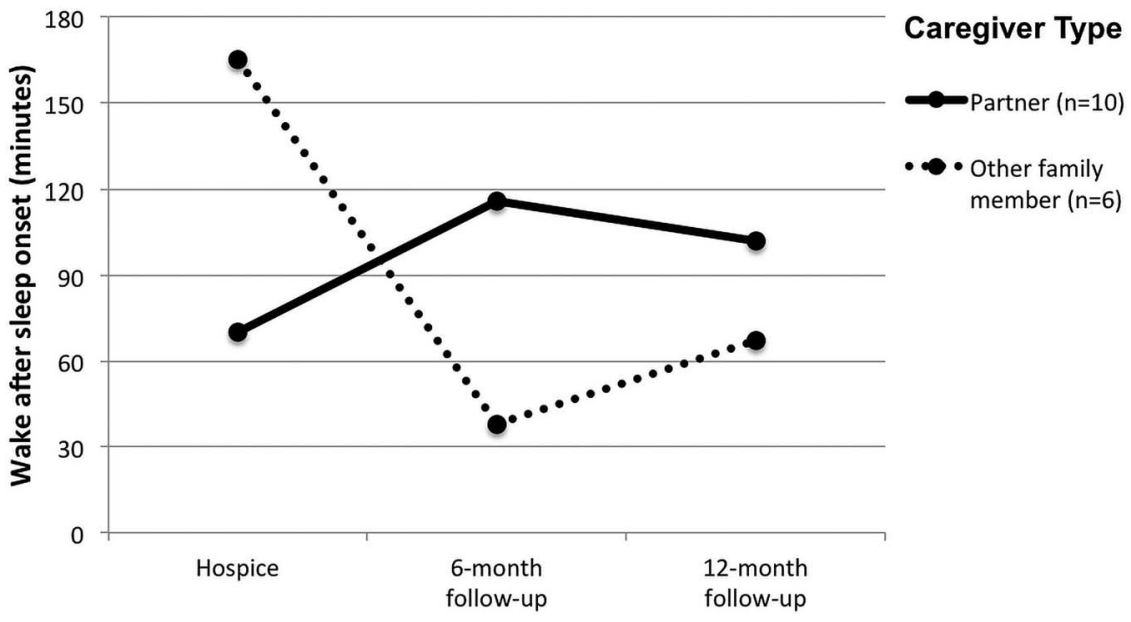

Time 
conflicts can affect family members during the caregiving period and in bereavement. Understanding the caregiver's sleep patterns, often characterised as 'sleeping with one eye open' when the patient is alive, is important for the caregivers' quality of life and perceived burden. This perspective extends into the bereavement period as well. ${ }^{1} 3782425$

Carter et $a l^{10}$ point out that a caregiver must often structure his or her life around the patient's needs. However, once the patient dies, that structure is lost and bereaved caregivers are vulnerable to insomnia, depression and complicated grief. Their results would suggest that the structure provided in cognitive-behavioural therapy may be an effective intervention for treating both the insomnia and depression commonly experienced by a bereaved family caregiver.

The results of this study should be considered in light of several limitations. The $50 \%$ enrolment rate and the $20 \%$ attrition rate suggest that this sample may not be representative of the larger population of caregivers of hospice patients. Strategies for improving participant enrolment and retention may need further consideration in a full-scale study of bereaved caregivers. Moreover, potential study participants experiencing significant distress were often not approached, which likely resulted in a sampling bias towards less distressed families. As such, these findings may underestimate sleep disturbance in this population. The patients and caregivers in this study were recruited while receiving care at a hospice, and the findings may differ for caregivers of patients receiving home care service. The study did not include a comparison group. In addition, actigraphy estimates are most reliable when sleep is monitored for 7 days or more, but to minimise participant burden, a shorter monitoring day was used, which may limit the reliability of the actigraphy estimates. Moreover, actigraphy estimates of sleep onset latency and sleep efficiency were not reported, and future studies should obtain more precise bed times and wake times so that these additional sleep parameters can be accurately estimated. Finally, the small sample size limits the generalisation of the study findings and precluded analysis of the effects of other caregiver characteristics (eg, gender, work status, living arrangement, other life stressors, comorbidities, medications and pre-existing symptoms/conditions), which may have influenced the caregiver's sleep. Depression often co-occurs with sleep disturbance, but the number of caregivers reporting depression in this small sample was insufficient to explore the relationship between sleep, depression and grief. Such factors need to be examined in future studies. A future study to explore these relationships in a larger sample is planned and has received ethics approval.

\section{CONCLUSION}

Family caregivers of hospice patients experience poor sleep quality, and this poor sleep generally extends at least through the first year of bereavement. However, the quality and quantity of sleep over time are influenced by caregiver characteristics such as age and relationship to the patient. Future studies are needed to identify other characteristics of caregivers at risk for poor sleep. A better understanding of these characteristics will help in the development and testing of tailored intervention strategies for this vulnerable population.

\section{Author affiliations}

${ }^{1}$ Department of Nursing Science, Institute of Health and Society, Faculty of Medicine, University of Oslo, Oslo, Norway

${ }^{2}$ Lovisenberg Diakonale Hospital, Oslo, Norway

${ }^{3}$ Lovisenberg Diakonale University College, Oslo, Norway

${ }^{4}$ Oslo and Akershus University College of Applied Sciences, Institute of Nursing, Oslo, Norway

${ }^{5}$ Department of Health, Sogn and Fjordane University College, Førde, Norway

${ }^{6}$ Department of Family and Health Care Nursing, University of California,

San Francisco, San Francisco, California, USA

${ }^{7}$ Department of Global Public Health and Primary Care, University of Bergen, Bergen, Norway

${ }^{8}$ Haukeland University Hospital, Norwegian Competence Center for Sleep Disorders, Bergen, Norway

Twitter Follow Anners Lerdal at @AnnersLerdal

Contributors AL, CLG, KAL and ES designed the study. AL, CLG, KAL, ES, EKG, APN and BB interpreted the data for the work. CLG and $A L$ analysed the data. Data was collected by ES. All the authors participated in revising the manuscript and approved the final version

Funding The study was funded from Lovisenberg Diakonale University College and Lovisenberg Diakonale Hospital.

Competing interests None declared.

Patient consent Obtained.

Ethics approval The study was approved by the Regional Medical Research Ethics Committee of Health East of Norway, the Norwegian Data Inspectorate, and Lovisenberg Hospice.

Provenance and peer review Not commissioned; externally peer reviewed.

Data sharing statement No additional data are available.

Open Access This is an Open Access article distributed in accordance with the Creative Commons Attribution Non Commercial (CC BY-NC 4.0) license, which permits others to distribute, remix, adapt, build upon this work noncommercially, and license their derivative works on different terms, provided the original work is properly cited and the use is non-commercial. See: http:// creativecommons.org/licenses/by-nc/4.0/

\section{REFERENCES}

1. Carter PA. Caregivers' descriptions of sleep changes and depressive symptoms. Oncol Nurs Forum 2002;29:1277-83.

2. Carter PA. A brief behavioral sleep intervention for family caregivers of persons with cancer. Cancer Nurs 2006;29:95-103.

3. Carter PA, Acton GJ. Personality and coping: predictors of depression and sleep problems among caregivers of individuals who have cancer. J Gerontol Nurs 2006;32:45-53.

4. Hearson B, McClement S, McMillan DE, et al. Sleeping with one eye open: the sleep experience of family members providing palliative care at home. J Palliat Care 2011;27:69-78.

5. Lerdal A, Gay CL, Saghaug E, et al. Sleep in family caregivers of patients admitted to hospice: a pilot study. Palliat Support Care 2014;12:439-44.

6. Meerlo P, Sgoifo A, Suchecki D. Restricted and disrupted sleep: effects on autonomic function, neuroendocrine stress systems and stress responsivity. Sleep Med Rev 2008;12:197-210.

7. von Kanel R, Mausbach BT, Ancoli-Israel S, et al. Sleep in spousal Alzheimer caregivers: a longitudinal study with a focus on the effects of major patient transitions on sleep. Sleep 2012;35:247-55. 
8. Chiu YW, Yin SM, Hsieh HY, et al. Bereaved females are more likely to suffer from mood problems even if they do not meet the criteria for prolonged grief. Psychooncology 2011;20:1061-8.

9. Chang EW, Tsai YY, Chang TW, et al. Quality of sleep and quality of life in caregivers of breast cancer patient. Psychooncology 2007;16:950-5.

10. Carter PA, Mikan SQ, Simpson C. A feasibility study of a two-session home-based cognitive behavioral therapy-insomnia intervention for bereaved family caregivers. Palliat Support Care 2009;7:197-206.

11. Buysse DJ, Reynolds CF III, Monk TH, et al. The Pittsburgh Sleep Quality Index: a new instrument for psychiatric practice and research. Psychiatry Res 1989;28:193-213.

12. Beck SL, Schwartz AL, Towsley G, et al. Psychometric evaluation of the Pittsburgh Sleep Quality Index in cancer patients. J Pain Symptom Manage 2004;27:140-8.

13. Pallesen $\mathrm{C}$, Nordhus $\mathrm{IH}$, Omvik $\mathrm{S}$, et al. Pittsburgh sleep quality index. J Norw Psych Assoc 2005;42:4.

14. Ancoli-Israel S, Cole R, Alessi C, et al. The role of actigraphy in the study of sleep and circadian rhythms. Sleep 2003;26:342-92.

15. Ferguson CJ. An effect size primer: a guide for clinicians and researchers. Prof Psychol Res Pract 2009;40:532-538.

16. Grov EK. The cancer trajectory-a model of phases. Vård i Norden 2014;34:2.
17. Davies B, Reimer JC, Brown P, et al. Fading away: the expereince of transition in families with terminal illness. New York: Baywood Publishing Company, Inc, 1995.

18. Exley C, Allen D. A critical examination of home care: end of life care as an illustrative case. Soc Sci Med 2007;65:2317-27.

19. Soothill K, Morris SM, Harman JC, et al. Informal carers of cancer patients: what are their unmet psychosocial needs? Health Soc Care Community 2001;9:464-75.

20. Wallace CL. Family communication and decision making at the end of life: a literature review. Palliat Support Care 2015;13:815-25.

21. Kissane DW, Bloch S, Dowe DL, et al. The Melbourne Family Grief Study, I: Perceptions of family functioning in bereavement. $A m \mathrm{~J}$ Psychiatry 1996;153:650-8.

22. Kissane DW, Bloch S, McKenzie M, et al. Family grief therapy: a preliminary account of a new model to promote healthy family functioning during palliative care and bereavement. Psychooncology 1998;7:14-25.

23. Kissane DW, McKenzie M, Bloch S, et al. Family focused grief therapy: a randomized, controlled trial in palliative care and bereavement. Am J Psychiatry 2006;163:1208-18.

24. Carter PA. Bereaved caregivers' descriptions of sleep: impact on daily life and the bereavement process. Oncol Nurs Forum 2005;32:741.

25. Hearson B, McClement S. Sleep disturbance in family caregivers of patients with advanced cancer. Int J Palliat Nurs 2007;13:495-501. 